\title{
Correction to: Promising Antibacterial Effects of Silver Nanoparticle-Loaded Tea Tree Oil Nanoemulsion: a Synergistic Combination Against Resistance Threat
}

\author{
Roqya Najafi-taher, ${ }^{1}$ Behnaz Ghaemi, ${ }^{1}$ Sharmin Kharrazi, ${ }^{1}$ Samira Rasoulikoohi, ${ }^{1,2}$ and Amir Amani ${ }^{1,3,4}$
}

Published online 11 January 2018

\section{Correction to: AAPS PharmSciTech} https://doi.org/10.1208/s12249-017-0922-y

With regrets, there is an error in the name of one of the authors which has only been noticed after publication.

"Sharmin Kharazi" should be corrected to "Sharmin Kharrazi".

The online version of the original article can be found at https:// doi.org/10.1208/s12249-017-0922-y

${ }^{1}$ Department of Medical Nanotechnology, School of Advanced Technologies in Medicine, Tehran University of Medical Sciences, Tehran, Iran.

${ }^{2}$ Antimicrobial Resistance Research Center, Rasoul-e-Akram Hospital, Iran University of Medical Sciences, Tehran, Iran.

${ }^{3}$ Medical Biomaterials Research Center (MBRC), Tehran University of Medical Sciences, Tehran, Iran.

${ }^{4}$ To whom correspondence should be addressed. (e-mail: aamani@tums.ac.ir) 